\title{
Analysis of Surface Roughness Heterogeneity and Scattering Behavior for Radar Measurements
}

\author{
Mehrez Zribi, Nicolas Baghdadi, and Christine Guérin
}

\begin{abstract}
The use of a theoretical backscatter model to analyze medium to low spatial resolution microwave data is still very complicated, particularly because of the difficulty in defining a unique roughness parameter, capable of adequately representing heterogeneous terrain. In this paper, an approach is proposed for roughness analysis and the modeling of backscattering, under conditions of surface heterogeneity. This paper is based on the use of a semiempirical backscattering model, defined with a single roughness parameter $\mathrm{Zs}=s^{2} / l$ ( $s$ being the root mean square surface height and $l$ the correlation length). The proposed backscattering model has been validated with integral equation model simulations, for high radar incidence angles, and within its domain of roughness validity. A range of experimental measurements was used to validate the model expressions. The effective low spatial resolution roughness, inferred from signals backscattered from a surface of heterogeneous roughness, is defined for different roughness classes.
\end{abstract}

Index Terms-Heterogeneity, modeling, roughness, surface scattering.

\section{INTRODUCTION}

$\mathbf{I}$ N RECENT years, various efforts have been devoted to the analysis of the backscattering characteristics of bare soils. Initially, different backscattering models (theoretical, semiempirical, and empirical) were developed [1]-[9]. More recently, several studies have proposed various approaches to improve roughness descriptions [10]-[13]. Such descriptions are essential to an accurate analysis and interpretation of backscattering behavior. Zribi et al. [12] introduced fractal and Brownian approaches, whereas Li et al. [14] proposed a general powerlaw description of roughness spectra. Zribi and Dechambre [15] later introduced a description based on the $\mathrm{Zs}$ parameter $[\mathrm{Zs}=$ $s^{2} / l$, where $s$ is the root mean square (rms) surface height and $l$ is the correlation length]. All of these descriptions have been applied to practical situations, at the scale of an agricultural field of homogenous roughness.

Simultaneously, the effectiveness of low-resolution spaceborne scatterometers for land-surface characterization has been demonstrated by a large number of studies related to vegetation, moisture, and roughness [16]-[20]. The questions addressed in

M. Zribi and C. Guérin are with the Centre d' Etude des Environments Terrestre et Planétaires, Centre National de la Recherche Scientifique, 78140 Vélizy, France (e-mail: zribi@cetp.ipsl.fr).

N. Baghdadi is with the Bureau de Recherches Géologiques et Minières, ARN/ATL, 45060 Orléans Cedex 2, France. this paper concern the applicability of a backscattering model, and the meaning of surface roughness in the case of large (scatterometer) footprints containing zones of different and heterogeneous roughness. Various authors have introduced fixed empirical parameters in order to fit backscattering models to their data [21], [22]. However, in some cases, the derived roughness values appear unrealistic when compared with in situ roughness measurements made at a local scale. Grippa and Woodhouse [23] analyzed the behavior of different backscattering models (geometrical optics (GEOs), physical optics (PHYs), small perturbation model (SPM), integral equation model (IEM), and the semiempirical model by Oh et al. $(\mathrm{OH})$ [3]) when used in the presence of heterogeneous soils. Only $\mathrm{OH}$ model expressions appear to incorporate heterogeneity. It is however important to note that although the rms height is factored into this model, variations in correlation length are not taken into account. Grippa and Woodhouse [23] proposed modified models that incorporate heterogeneity by means of a probability distribution. However, this approach requires other hypothesis, particularly for the roughness probability distribution.

For these reasons, the principal objective of this paper is to reveal the relationship between effective roughness at a low spatial resolution and radar backscattering, in the case of bare soils. Analytical backscattering models such as IEM, which require several roughness parameters as input (rms height, correlation length, surface spectrum) are very difficult to use for the analysis of such relationships. Furthermore, Grippa and Woodhouse [23] have shown that these models are not suitable for retrieving surface characteristics from a heterogeneous area. For example, as discussed in the same paper, backscattering depends quadratically on the rms height for SPM. Consequently, if the correlation length is constant, the rms height corresponding to the sum of backscattering from two surfaces with two different rms heights $\left(s_{\mathrm{A}}, s_{\mathrm{B}}\right)$ could be computed as $s=\sqrt{\left(s_{\mathrm{A}}^{2}+s_{\mathrm{B}}^{2}\right) / 2}$.

In order to overcome the difficulties encountered when using analytical models with different roughness input parameters, it is proposed in this paper to develop a "semiempirical" model that is characterized by a single surface roughness parameter, and which remains in agreement with the IEM model. There is a strong need for improved clarity in the discussion of the relationship between low spatial resolution roughness (including heterogeneous roughness) and backscattering.

This paper is organized into five sections, of which Section II presents the semiempirical model developed by the authors, and discusses its validation using the IEM model. Section III describes the comparison between the developed model and 


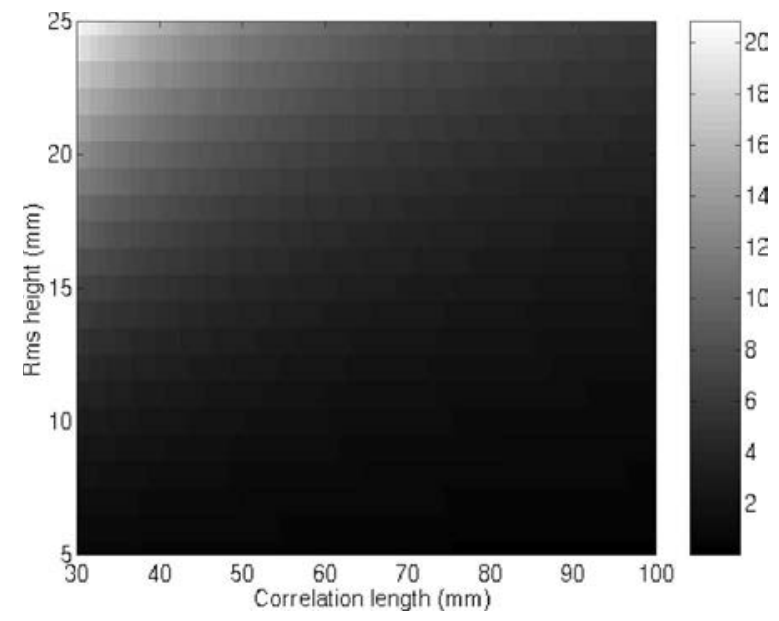

Fig. 1. Illustration of the parameter $\mathrm{Zs}$ as a function of rms height and correlation length.

experimental radar data obtained with different types of roughness, and Section IV analyzes the concept of an effective low spatial resolution roughness computed from heterogeneous roughness values. Finally, the authors' conclusions are presented in Section V.

\section{Semiempirical Model DeVElopment}

\section{A. Analysis of the Relationship Between Backscattering Signal and the Parameter Zs}

The characterization of surface roughness is one of the key requirements in achieving correct analysis of backscattering behavior. It is noteworthy that an increase in the number of surface roughness parameters in a model also increases the difficulty of data inversion. In fact, in the case of some specific radar configurations, the use of more than two roughness parameters, in addition to a surface dielectric constant, renders the inversion inaccurate. Zribi and Dechambre [15] proposed the introduction of the parameter $\mathrm{Zs}=s^{2} / l$, combining the effects of rms height and correlation length. This is in effect the product of the rms height $s$, related to the power of the surface height variations, with the ratio $s / l$, which represents a slope factor. The underlying idea is to introduce the influence of a slope, which is an important soil feature in estimating $\sigma^{0}$. Small values of Zs correspond to small values of $s$ and/or large values of $l$, whereas large values of Zs correspond to large values of $s$ or small values of $l$. A smooth soil surface (without clods) is generally characterized by a small value of $s$ and a medium to large value of $l$, thus to a small value of Zs. Ploughed soil often corresponds to a large value of $s$ and to a medium to large value of $l$, thus to a medium to large value of Zs. Very cloddy soil, even with a small $s$, is characterized by a very small value of $l$ and thus leads to large values of Zs. Fig. 1 provides values of Zs computed for a large range of rms soil heights and correlation lengths. In view of the very strong correlation demonstrated by Zribi and Dechambre [15] between backscattered radar signals and $\mathrm{Zs}$, the latter has been chosen as the unique roughness parameter to be used in the model proposed in this paper.

\section{B. Proposed Semiempirical Model}

In order to analyze the effect of surface roughness on the backscattering behavior for different spatial resolutions, it is very important, as noted in the Introduction, to use a backscattering model with just one roughness parameter. For this reason, our objective in this section is to propose a new semiempirical model dependent on a single roughness parameter Zs. The proposed model is based on a complete separation between roughness and moisture effects, a hypothesis that has been found to be robust in various theoretical and experimental studies [5], [24]. The semiempirical model is adjusted to fit the IEM single backscattering model [2], [25] written as

$$
\begin{aligned}
\sigma_{\mathrm{pp}}^{0} & =\frac{k^{2}}{2} \exp \left[-2 k_{z}^{2} s^{2}\right] \sum_{n=1}^{\infty} s^{2 n}\left|I_{\mathrm{pp}}^{n}\right|^{2} \frac{W^{(n)}\left(-2 k_{x}, 0\right)}{n !} \\
p & =h, v
\end{aligned}
$$

where $k_{z}=k \cos \theta, k_{x}=k \sin \theta, \theta$ is the radar incidence angle, $k$ is the wavenumber, and $s$ is the rms surface height. $I_{\mathrm{pp}}^{n}$ is a function of the radar incidence angle, the dielectric constant of the soil $s$, and the Fresnel reflection coefficient. $W^{(n)}\left(-2 k_{x}, 0\right)$ is the Fourier transform of the $n$th power of the surface correlation function.

Although the theoretical domain of validity of the above expression is generally limited to ks $<3$ [26], various experimental studies [27], [28] have shown that the single-scattering model is limited to approximately ks $<1.2$. Therefore, in the model presented here, surface roughness will be limited to smooth and medium values $(\mathrm{ks}<1.2)$. It is important to note that this is the domain in which a roughness-induced backscattering exhibits the strongest dynamic range, since at higher roughness values, the radar signal approaches saturation. In this paper, the developed model is limited to high incidence angles only $\left(\theta>35^{\circ}\right)$; at lower angles the single roughness parameter representation would be inadequate, since new effects, resulting from low roughness frequencies, would not be accounted for.

The proposed model is written as

$$
\begin{aligned}
\sigma_{0}^{\mathrm{vv}} & =0.5(k \mathrm{Zs})^{0.84} \frac{\cos \theta^{4}}{\sin \theta^{3}} \alpha_{\mathrm{vv}}^{2} \\
\sigma_{0}^{\mathrm{hh}} & =3.2110^{p(\theta)}(k \mathrm{Zs})^{q(\theta)} \frac{\cos \theta^{4}}{\sin \theta^{3}} \alpha_{\mathrm{hh}}^{2}
\end{aligned}
$$

with

$$
\begin{aligned}
& \alpha_{\mathrm{hh}}=R_{\perp}=\frac{\cos \theta-\sqrt{\varepsilon_{\mathrm{r}}-\sin ^{2} \theta}}{\cos \theta+\sqrt{\varepsilon_{\mathrm{r}}-\sin ^{2} \theta}} \\
& \alpha_{\mathrm{vv}}=\left(\varepsilon_{\mathrm{r}}-1\right) \frac{\sin ^{2} \theta-\varepsilon_{\mathrm{r}}\left(1+\sin ^{2} \theta\right)}{\left[\varepsilon_{\mathrm{r}} \cos \theta+\sqrt{\varepsilon_{\mathrm{r}}-\sin ^{2} \theta}\right]^{2}}
\end{aligned}
$$

$R_{\perp} \quad$ Fresnel coefficient for horizontal $(\mathrm{HH})$ polarization;

$\varepsilon_{\mathrm{r}} \quad$ relative dielectric constant;

$k$ wavenumber;

$\theta \quad$ incidence angle.

$$
\begin{aligned}
& q(\theta)=2.6289 \theta^{2}-3.2561 \theta+1.969 \\
& p(\theta)=2.303 \theta^{2}-2.3217 \theta
\end{aligned}
$$
with $\theta$ in radians with $\theta$ in radians. 
The expressions given in (2) and (3) were derived as follows. The SPM has been used, allowing roughness and moisture effects to be separated.

The backscattering coefficient is written as

$$
\sigma_{0}=g(R, \theta) \cdot f(\varepsilon, \theta)
$$

and the roughness component is given by

$$
g=k^{4} s^{2} W(2 k \sin \theta, 0) .
$$

In general, exponential correlation function is used to describe natural soil surfaces, thus leading to

$$
g=k^{4} s^{2} \frac{l^{2}}{\left(1+(2 k l \sin \theta)^{2}\right)^{1.5}} .
$$

By taking $(2 k l \sin \theta) \gg 1$, the above expression for $g$ can be simplified to

$$
g \approx \frac{k}{8} \frac{s^{2}}{l \sin ^{3} \theta}=\frac{k \mathrm{Zs}}{8 \sin ^{3} \theta} .
$$

This hypothesis is particularly useful for high incidence angles. With a view to adjusting the proposed model to the IEM, the roughness component is expressed here as a function of Zs. As simulations show that the SPM and IEM models have approximately the same behavior as a function of surface moisture, it is proposed to adjust the model presented here to the IEM by considering two variables: a parameter $(a)$ used to define the power law of $(k \mathrm{Zs})$, and a proportionality constant $(b)$

$$
g=b \frac{(k \mathrm{Zs})^{a}}{\sin ^{3} \theta} .
$$

The proposed semiempirical model depends only on the Zs parameter to describe surface roughness. This limitation to one parameter is very interesting for radar measurement inversion. However, it is important to note that our hypotheses concerning the correlation function shape or the expression of the model limit necessarily the validity domain of the model, particularly for high radar frequencies.

\section{Comparison With IEM Simulations}

In order to validate the proposed model, use of the IEM has been restricted to its domain of validity $(\mathrm{ks}<1.2)$. Simulations were made for a large range of surface roughness parameters. Heterogeneous roughness parameters were generated using a Monte Carlo approach, based on uniform rms height $(0.3-1.2 \mathrm{~cm})$ and correlation length $(3-20 \mathrm{~cm})$ distributions. Three hundred IEM simulations were made using an exponential correlation function with constant soil moisture (30\%). Fig. 2 compares the results predicted by the IEM and proposed models, for $\mathrm{HH}$ and vertical (VV) polarizations in C-band, using four different incidence angles $35^{\circ}, 40^{\circ}, 45^{\circ}$, and $50^{\circ}$. Good agreement is found between the two models; in particular, for the VV polarization, the rms error is less than $0.26 \mathrm{~dB}(0.25 \mathrm{~dB}$ for $35^{\circ}, 0.23 \mathrm{~dB}$ for $40^{\circ}, 0.24 \mathrm{~dB}$ for $45^{\circ}$, and $0.26 \mathrm{~dB}$ for $50^{\circ}$ ).
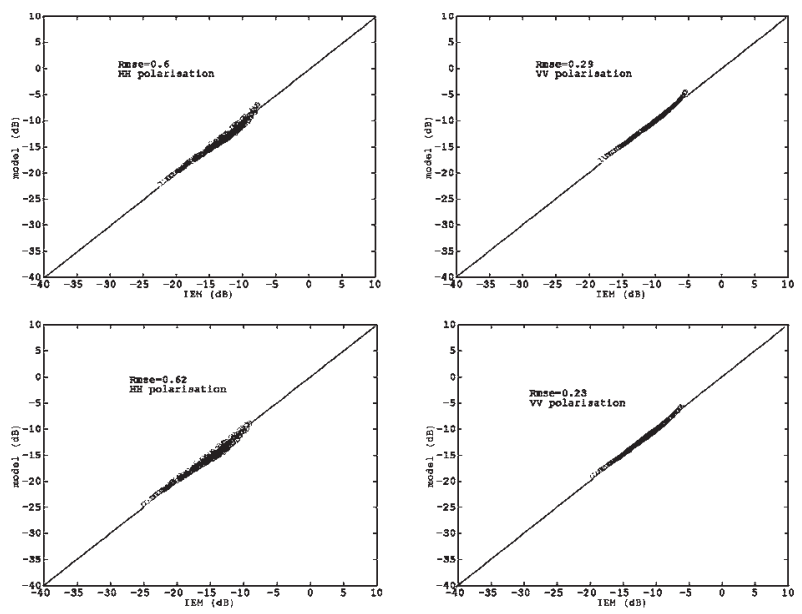

(b)
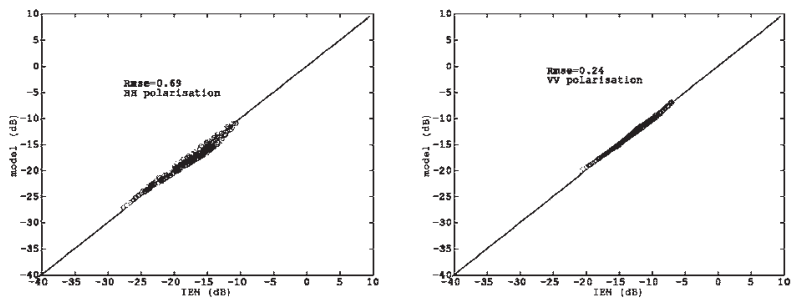

(c)
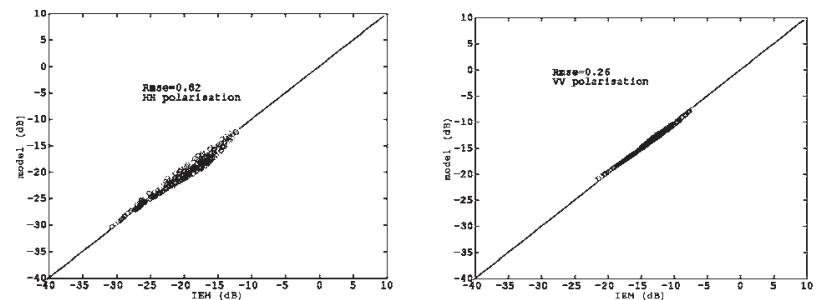

Fig. 2. Comparison between backscattering predictions from the IEM and the proposed semiempirical model for $\mathrm{HH}$ and $\mathrm{VV}$ polarizations, for different incidence angles: (a) $35^{\circ}$, (b) $40^{\circ}$, (c) $45^{\circ}$, and (d) $50^{\circ}$.

TABLE I

RMS ERrors Between IEM and Proposed Model Simulations, FOR DIFFERENT CONFIGURATIONS OF MOISTURE (Mv) AND INCIDENCE ANGLE $(\theta)$

\begin{tabular}{|l|l|l|l|l|l}
\hline \multicolumn{2}{|c|}{} & $\Theta=35^{\circ}$ & $\Theta=40^{\circ}$ & $\Theta=45^{\circ}$ & $\Theta=50^{\circ}$ \\
\hline \multirow{2}{*}{$\begin{array}{l}\mathrm{Mv}= \\
10 \%\end{array}$} & $\mathrm{HH}$ & 0.47 & 0.69 & 1 & 1.6 \\
\cline { 2 - 6 } & $\mathrm{VV}$ & 0.29 & 0.29 & 0.28 & 0.27 \\
\hline \multirow{2}{*}{$\begin{array}{l}\mathrm{Mv}= \\
20 \%\end{array}$} & $\mathrm{HH}$ & 0.53 & 0.56 & 0.66 & 0.84 \\
\cline { 2 - 6 } & $\mathrm{VV}$ & 0.26 & 0.25 & 0.25 & 0.26 \\
\hline $\mathrm{Mv}=$ & $\mathrm{HH}$ & 0.6 & 0.62 & 0.69 & 0.82 \\
\cline { 2 - 6 } $30 \%$ & $\mathrm{VV}$ & 0.25 & 0.23 & 0.24 & 0.26 \\
\hline
\end{tabular}

For $\mathrm{HH}$ polarization, the rms error does not exceed $0.82 \mathrm{~dB}$ $\left(0.6 \mathrm{~dB}\right.$ for $35^{\circ}, 0.62 \mathrm{~dB}$ for $40^{\circ}, 0.69 \mathrm{~dB}$ for $45^{\circ}$, and $0.82 \mathrm{~dB}$ for $50^{\circ}$ ). This result convincingly supports the assumption that Zs can replace all the roughness parameters used in the IEM model, and justifies the use of the proposed model to analyze roughness behavior for the proposed domain of validity.

In order to characterize moisture effects, the same function can be used as that derived for the SPM model. Table I illustrates the good degree of coherence between IEM and proposed model simulations, where an rms error less than $1 \mathrm{~dB}$ is found (except for one case) over a large range of soil moistures. 


\section{ANALysis BASED ON Experimental RADAR DATA}

In the previous section, the coherence between the IEM and proposed model has been demonstrated. The purpose of this section is to compare the roughness component behavior predicted by the present model with that found in experimental radar data.

\section{A. Database Description}

For this paper, data acquired over agricultural watersheds in France were used [9], [15]. Radar soundings were carried out at high incidence angles, all in $\mathrm{HH}$ polarization, by various active orbiting sensors (ASAR/ENVISAT, RADARSAT-, SIR-C). The following three experimental campaigns are considered in this paper.

1) Orgeval (1994): The Orgeval watershed is located in the east of Paris, France. The soil texture is approximately constant over the whole basin: $17 \%$ clay, $78 \%$ silt, and $5 \%$ sand. The Orgeval (1994) radar data were acquired with the SIR-C radar.

2) Pays de Caux (1998): The test site is the Blosseville watershed located in the Pays de Caux in northern France. The soil is characterized by a loamy texture (13\% clay, $60 \%$ silt, and $22.4 \%$ sand). The radar data were acquired by RADARSAT-I.

3) Villamblain (2003): The Villamblain site is located in the center of France with a very flat topography. The site is characterized by soils composed of about $60 \%$ loam, $30 \%$ clay, and $10 \%$ sand. The data were acquired by the ASAR radar instrument on ENVISAT.

Simultaneously to the above-described radar acquisitions, ground measurements were carried out in a large number of test fields. The soil moisture was measured within the upper $5 \mathrm{~cm}$ using a gravimetric method and/or a time-domain reflectometry (TDR) probe. All measurements used in this paper correspond to high moisture values (higher than $30 \%$ for volumetric moisture). Therefore, in the following discussions, the moisture levels are considered to be approximately constant. Roughness measurements were made using a pin profiler (total length of 1 and $2 \mathrm{~m}$ and a resolution of 5 and $10 \mathrm{~mm}$, respectively). In order to guarantee sufficient statistical accuracy, ten profiles were measured for each test field. For the full set of measurements, the rms height ranges between 0.38 and $2.5 \mathrm{~cm}$, the correlation length varies between 2.5 and $12 \mathrm{~cm}$ and the corresponding parameter Zs ranges between 0.016 and $2.64 \mathrm{~cm}$. Roughness values are illustrated in Fig. 3.

\section{B. Validation of the Model Behavior Within Its Validity Domain}

In this section, it is shown that the semiempirical model provides simulated backscattering results in good agreement with experimental radar data.

In the case of the HH polarization, the backscattering signal could be written as

$$
\sigma_{0}^{\mathrm{hh}}[\mathrm{dB}]=10 \log (g(R, \theta))+10 \log (f(\varepsilon, \theta))
$$

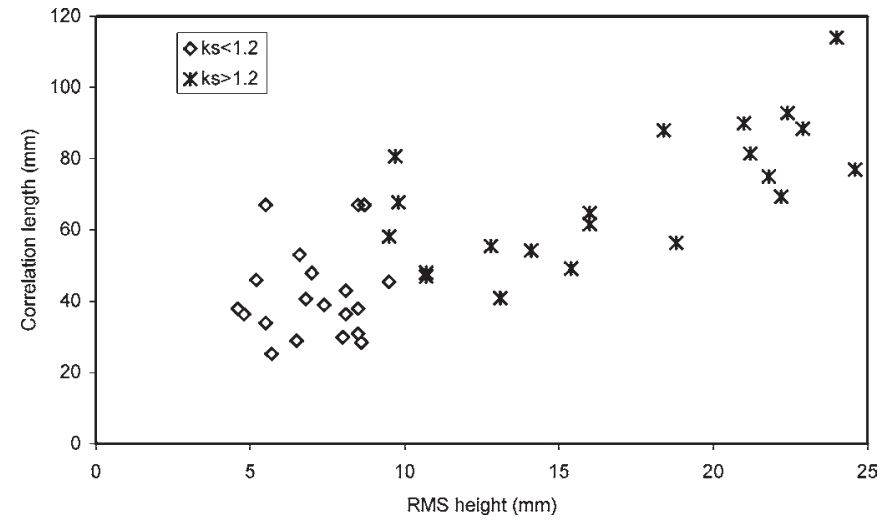

Fig. 3. Illustration of roughness measurements of different test fields used in the proposed model validation.

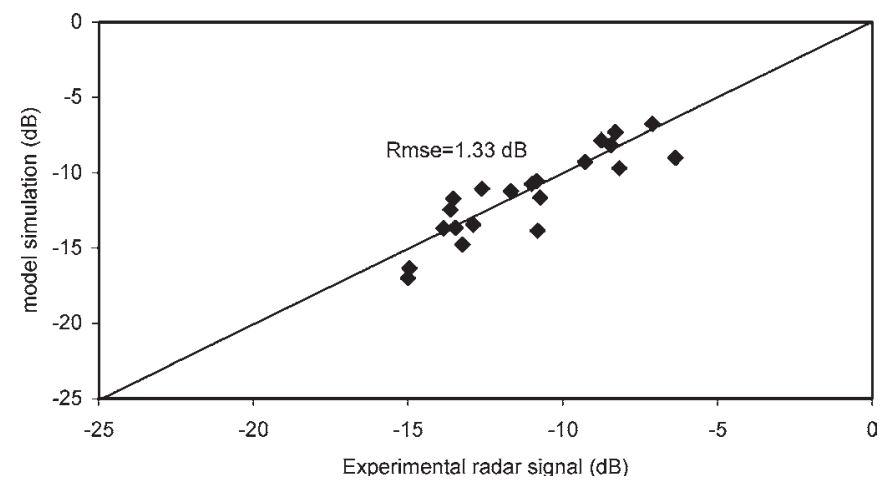

Fig. 4. Comparison between experimental radar data and simulations using the proposed model, within its validity domain $(\mathrm{ks}<1.2)$ for various incidence angles and $\mathrm{HH}$ polarization.

where

$$
g=\left((k \mathrm{Zs})^{q(\theta)}\right) .
$$

For the purposes of the present discussion, soil moisture is assumed to be almost constant (higher than $30 \%$ ), and analysis of the results is centered principally around the influence of soil roughness on backscattering.

Fig. 4 provides a comparison between experimental radar data and model simulations for 21 different test fields, for which measured roughness respects the domain of validity $(\mathrm{ks}<1.2)$ of the semiempirical model, as illustrated in Fig. 3. The data correspond to various incidence angles $\left(37.4^{\circ}, 39^{\circ}, 40^{\circ}, 44^{\circ}, 47^{\circ}\right)$ in $\mathrm{HH}$ polarization. The rms error between experimental and predicted signals is equal to $1.33 \mathrm{~dB}$, thus demonstrating that the use of a single roughness parameter can provide reliable results of good quality. Concerning the soil moisture variation effect on the proposed model, it is in good agreement with IEM model behavior as it is illustrated in Table I.

\section{Analysis of the Model Behavior Outside the Bounds of Its Validity Domain}

In this section, experimental radar data are compared with results derived from the semiempirical model, for roughness values outside its limits of validity (ks $>1.2)$. Fig. 5 compares, as in Fig. 4, radar data with results predicted by the model, 


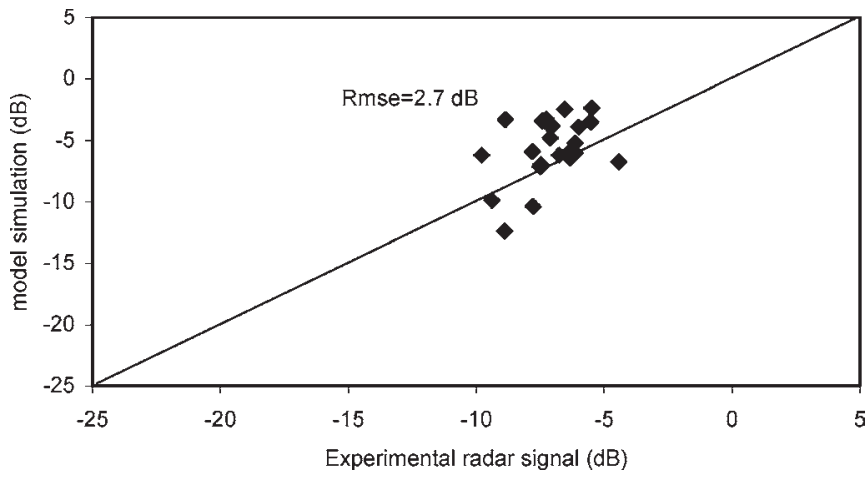

Fig. 5. Comparison between experimental radar data and simulations using the proposed model for high roughness $(\mathrm{ks}>1.2)$ and various incidence angles and $\mathrm{HH}$ polarization.

for a range of incidence angles. In this case, a discrepancy is observed between data and simulations, with an rms error of $2.7 \mathrm{~dB}$. The model is seen to overestimate the backscattered signal, a result generally observed with the IEM model for high surface roughness [28]. In practice, the radar data saturates more quickly than the signal levels predicted by the backscattering model.

\section{Characterization OF Low Spatial RESOLUTION ROUGHNESS}

\section{A. Analysis Within the Model's Domain of Validity}

In this section, the semiempirical model is used to analyze the impact of heterogeneous roughness on low spatial resolution data.

The incoherent sum of signals backscattered by $n$ fields is written as

$$
\sigma_{0}^{\text {low }}=\sum_{n} c_{i} \sigma_{0}^{i}\left(s_{i}, l_{i}, \varepsilon_{i}\right)
$$

where

$c_{i} \quad$ signal fraction corresponding to the $i$ th field;

$\sigma_{0}^{i} \quad$ backscattered signal from the $i$ th field;

$s_{i} \quad$ rms height of the $i$ th field;

$l_{i} \quad$ correlation length of the $i$ th field;

$\varepsilon_{i} \quad$ dielectric constant of the $i$ th field.

Assuming constant soil moisture content for all fields and combining (2), (3), and (9), a low-roughness parameter Zs can be written as

$$
\begin{aligned}
& \mathrm{Zs}_{\text {low }}=\left(\sum_{n} c_{i} \mathrm{Zs}_{i}^{0.84}\right)^{1 / 0.84} \quad \text { for } \mathrm{VV} \text { polarization } \\
& \mathrm{Zs}_{\text {low }}=\left(\sum_{n} c_{i} \mathrm{Zs}_{i}^{q(\theta)}\right)^{1 / q(\theta)} \quad \text { for } \mathrm{HH} \text { polarization. }
\end{aligned}
$$

These equations provide relationships between the Zs parameter for $n$ different local fields, and the effective low spatial resolution parameter $\mathrm{Zs}_{\text {low }}$ of the combined fields. Equation (11) shows that for $\mathrm{HH}$ polarization, the effective $\mathrm{Zs}$ for heterogeneous surfaces is dependent on incidence angle only.

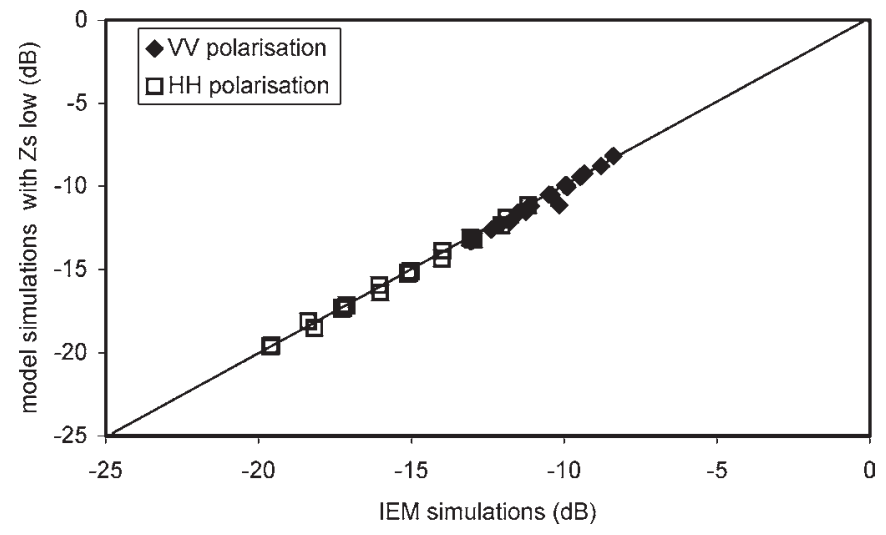

Fig. 6. Comparison between an incoherent sum of IEM simulations computed for heterogeneous roughness cases, and simulations from the semiempirical model using effective roughness values for the parameter $\mathrm{Zs}$ for four incidence angles $\left(35^{\circ}, 40^{\circ}, 45^{\circ}\right.$, and $\left.50^{\circ}\right)$.

The principal objective of this paper is to provide an analysis of radar backscattering behavior as a function of heterogeneous roughness at low spatial resolutions. Having defined effective low spatial resolution roughness, the authors undertook to compare IEM simulations with the backscattering behavior predicted by the proposed model, using various surfaces of heterogeneous roughness.

For each of 300 different cases, the incoherent sum of IEM model simulations for $n$ fields with different roughness characteristics was compared with the developed model using the effective Zs parameter computed from (10) or (11). In order to correctly validate the proposed model at low spatial resolution, it was chosen to consider different types of roughness distribution to represent surface heterogeneity: For each different case, various types of roughness distribution were considered (uniform, Gaussian, Weibull, etc.). Fig. 6 shows the outcome of these comparisons for four incidence angles $\left(35^{\circ}, 40^{\circ}, 45^{\circ}\right.$, and $\left.50^{\circ}\right)$, thus demonstrating that a good degree of coherence exists between the two methods.

This result confirms the potential of an effective Zs parameter in describing low spatial resolution roughness, when used with the proposed semiempirical model to simulate low spatial resolution backscattering.

After the analysis made with IEM model, a comparison is made between the proposed semiempirical model and experimental radar data in $\mathrm{HH}$ polarization. Each datum corresponds to the radar signal computed from three or four fields with a different surface roughness. Table II illustrates the values of roughness (rms height, correlation length) for each datum. $\mathrm{Zs}_{\text {low }}$ (representing the heterogeneous roughness for each datum) is computed using (11) with roughness measurements for each field. Fig. 7 illustrates this comparison with six different data (corresponding to 21 different fields). It shows a good agreement between data and model with an rms error equal to $0.47 \mathrm{~dB}$.

\section{B. Discussion of the Model Outside Its Range of Validity}

The study with the semiempirical model is made for fields with a surface roughness ranging between smooth and medium. When a greater range of surface types is present (smooth, 
TABLE II

ILLUSTRATION OF ROUGHNESS PARAMETERS (RMS HEIGHT, Correlation LENGTHS) MEASURED IN THE Six DiFFERENT HETEROGENEOUS SOIL SURFACES USED FOR COMPARISON BetweEn Model Simulation ANd Radar Data

\begin{tabular}{l|l|l|}
\hline data & $\begin{array}{l}\text { Number of } \\
\text { fields }\end{array}$ & $\begin{array}{l}\text { Fteld roughness parameters }(s, 1) \text { composing heterogeneous } \\
\text { surface }\end{array}$ \\
\hline 1 & 3 & $(0.7,4.8),(0.52,4.6),(0.55,3.45)$ \\
\hline 2 & 3 & $(0.85,6.7),(0.68,4.1),(0.46,3.8)$ \\
\hline 3 & 3 & $(0.81,3.7),(0.52,4.6),(0.55,3.4)$ \\
\hline 4 & 4 & $(0.87,6.7),(0.95,4.6),(0.65,2.9),(0.48,3.64)$ \\
\hline 5 & 4 & $(0.86,2.85),(0.57,2.6),(0.81,4.3),(0.74,3.9)$ \\
\hline & 3 & $(0.85,3.1),(0.78,3.1),(0.85,3.8)$ \\
\hline
\end{tabular}

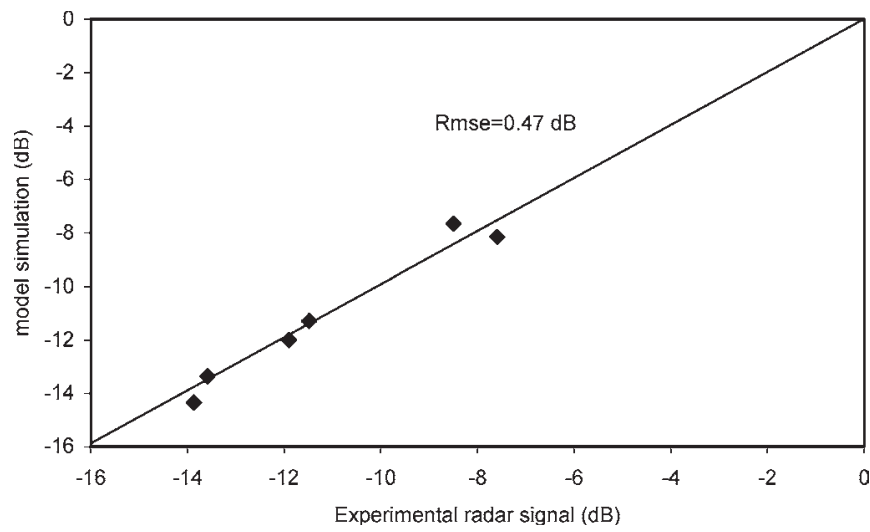

Fig. 7. Comparison between experimental radar data and simulations using the proposed model for low-resolution data with heterogeneous roughness and $\mathrm{HH}$ polarization.

medium, and rough), the relationship between low spatial resolution roughness and field scale roughness becomes more complicated. If different powers are used for different degrees of roughness, the effective low spatial resolution parameter Zs can then be written as

$$
\mathrm{Zs}_{\text {low }}=\left(\sum_{n} c_{i} \mathrm{Zs}_{i}^{B_{i}(\theta)}\right)^{1 / B_{t}(\theta)}
$$

where $B_{i}(\theta)$ is the power corresponding to each type of roughness.

For low spatial resolution data with topographic (local angle) variations, the effective Zs parameter must also take into account variations in incidence angle. With the mean local angle taken to be zero, the effective $\mathrm{Zs}$ parameter for $\mathrm{VV}$ polarization can then be written as

$$
\mathrm{Zs}_{\mathrm{low}}=\left[\left(\sum_{n} c_{i} \mathrm{Zs}_{i}^{0.84} \frac{\cos \theta_{i}^{4}}{\sin \theta_{i}^{3}} \alpha_{\mathrm{vv} i}^{2}\right) /\left(\frac{\cos \theta^{4}}{\sin \theta^{3}} \alpha_{\mathrm{vv}}^{2}\right)\right]^{1 / 0.84}
$$

where

$\theta \quad$ mean incidence angle;

$\theta_{i} \quad \theta-\theta_{\text {local }}$

$\theta_{\text {local }}$ local angle of the $i$ th field.

The preceding discussions show that even for low-resolution radar data, the effective roughness parameter $Z s$ has a distinct physical meaning, which is closely linked to the local roughness characteristics of the terrain under study.

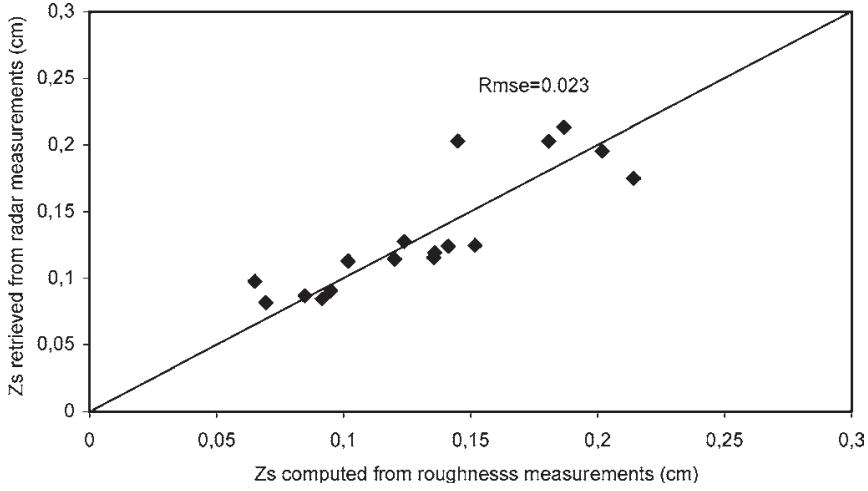

Fig. 8. Comparison between retrieved roughness from radar measurements and coarse-resolution roughness computed from real measurements over different homogenous soil surfaces.

\section{Inversion Model}

After the validation of our model to simulate radar signal over heterogeneous soil surfaces, we propose to apply the inversion model to retrieve surface roughness from radar measurements over heterogeneous surfaces. For our case, radar data exist only in $\mathrm{HH}$ polarization with one incidence angle for each experimental campaign. Therefore, radar inversion is made only to calculate roughness parameter $\mathrm{Zs}$ and not moisture, which is considered as known (measured). We consider 17 data with for each case a heterogeneous soil with three or four different agricultural fields. Fig. 8 illustrates the comparison between retrieved roughness from radar data (with incidence angle raging between $37.4^{\circ}$ and $47^{\circ}$ ) using our model and roughness computed from the three or four measured soil roughness of each field, using (11). We observe a good agreement with an rms error equal to $0.023 \mathrm{~cm}$. This result confirms the potential of this model to retrieve heterogeneous surface roughness in spite of its validity domain limitation for high radar frequencies.

\section{CONCLUSION}

The objective of this paper is to analyze the role of roughness, for the case of heterogeneous terrains, in a surface scattering model. A semiempirical model is proposed, which clearly distinguishes between roughness and moisture effects. Roughness is described with just one parameter $\mathrm{Zs}=s^{2} / l$. The proposed model has been validated by comparing its predictions with those obtained with the IEM single-scattering model. Good agreement is found between these models for high incidence angles $\left(\theta>35^{\circ}\right)$ and for small or medium degrees of roughness (ks $<1.2$ ). A comparison made with experimental radar data shows that model predictions and observed data are in good agreement, for homogenous surfaces with ks $<1.2$. For a terrain characterized by a high degree of roughness, the model is found to overestimate the backscattering coefficient. In the case of heterogeneous terrains observed with low spatial resolution, the proposed model allows a single value of effective surface roughness to be used. For a case study of $n$ fields with heterogeneous roughness characteristics, the backscattering predictions obtained with the semiempirical model are shown to be well correlated with the result obtained by incoherently summing $n$ IEM simulations and experimental radar data. The effective 
roughness of a heterogeneous area is defined for different types of surface roughness, for both $\mathrm{HH}$ and $\mathrm{VV}$ polarizations. The comparison between computed heterogeneous roughness over different test sites and retrieved roughness using inversion model and radar data shows a good agreement.

The proposed model with a limited roughness validity domain is certainly more adapted to low frequencies up to C-band.

Further studies will propose a global mapping of the $\mathrm{Zs}$ parameter, based on data obtained from existing low resolution microwave sensors, in particular ASAR (ENVISAT) and the European Remote Sensing Satellite 2 or the Advanced Scatterometer wind scatterometer.

\section{ACKNOWLEDGMENT}

The authors would like to thank the European Space Agency for having providing them with ASAR images free of charge, through project number 351 ENVISAT/ASAR. The authors would like to thank also all the team for their logistic support in terrain campaigns.

\section{REFERENCES}

[1] F. T. Ulaby, R. K. Moore, and A. K. Fung, Microwave Remote Sensing Active and Passive. Norwood, MA: Artech House, 1986.

[2] A. K. Fung, Z. Li, and K. S. Chen, "Backscattering from a randomly rough dielectric surface," IEEE Trans. Geosci. Remote Sens., vol. 30, no. 2, pp. 356-369, Mar. 1992.

[3] Y. Oh, K. Sarabandi, and F. T. Ulaby, "An empirical model and an inversion technique for radar scattering from bare soil surfaces," IEEE Trans. Geosci. Remote Sens., vol. 30, no. 2, pp. 370-381, Mar. 1992.

[4] _ _ "An inversion algorithm to retrieve soil moisture and surface roughness from polarimetric radar observations," in Proc. IGARSS, Pasadena, CA, Aug. 8-12, 1994, vol. 3, pp. 1582-1584.

[5] P. C. Dubois, J. Van Zyl, and T. Engman, "Measuring soil moisture with imaging radars," IEEE Trans. Geosci. Remote Sens., vol. 33, no. 4, pp. 915-926, Jul. 1995.

[6] J. Shi, J. Wang, A. Y. Hsu, P. E. O'Neill, and T. Engmann, "Estimation of bare surface soil moisture and surface roughness parameter using L-Band SAR image data," IEEE Trans. Geosci. Remote Sens., vol. 35, no. 5, pp. 1254-1265, Sep. 1997.

[7] S. Le Hégarat-Mascle, M. Zribi, F. Alem, A. Weisse, and C. Loumagne, "Soil moisture estimation from ERS/SAR data: Toward an operational methodology," IEEE Trans. Geosci. Remote Sens., vol. 40, no. 12, pp. 1-12, Dec. 2002.

[8] Y. Oh, "Quantitative retrieval of soil moisture content and surface roughness from multipolarized radar observations of bare soil surfaces," IEEE Trans. Geosci. Remote Sens., vol. 42, no. 3, pp. 596-601, Mar. 2004.

[9] N. Baghdadi, N. Holah, and M. Zribi, "Soil moisture estimation using multi-incidence and multi-polarization ASAR SAR data," Int. J. Remote Sens., vol. 27, no. 10, pp. 1907-1920, 2006.

[10] F. Mattia and T. Le Toan, "Backscattering properties of multi-scale rough surfaces," J. Electromagn. Waves Appl., vol. 13, no. 4, pp. 491-526, 1999.

[11] M. W. J. Davidson, T. Le Toan, F. Mattia, G. Satalino, T. Manninen, and M. Borgeaud, "On the characterization of agricultural soil roughness for radar remote sensing studies," IEEE Trans. Geosci. Remote Sens., vol. 38, no. 2, pp. 630-640, Mar. 2000.

[12] M. Zribi, V. Ciarletti, and O. Taconet, "Validation of a rough surface model based on fractional Brownian geometry with SIRC and ERASME radar data over Orgeval site," Remote Sens. Environ., vol. 73, no. 1, pp. 65-72, 2000.

[13] N. Baghdadi, I. Gherboudj, M. Zribi, M. Sahebi, F. Bonn, and C. King, "Semi-empirical calibration of the IEM backscattering model using radar images and moisture and roughness field measurements," Int. J. Remote Sens., vol. 25, no. 18, pp. 3593-3623, Sep. 2004.

[14] Q. Li, J. C. Shi, and K. S. Chen, "A generalized power law spectrum and its applications to the backscattering of soil surfaces based on the integral equation model," IEEE Trans. Geosci. Remote Sens., vol. 40, no. 2, pp. 271-281, Feb. 2002.

[15] M. Zribi and M. Dechambre, "An new empirical model to retrieve soil moisture and roughness from radar data," Remote Sens. Environ., vol. 84, no. 1 , pp. $42-52,2002$.
[16] P. L. Frison and E. Mougin, "Use of ERS-1 wind scatterometer data over land surfaces," IEEE Trans. Geosci. Remote Sens., vol. 34, no. 2, pp. 550-560, Mar. 1996.

[17] W. Wagner, G. Lemoine, M. Borgeaud, and H. Rott, "A study of vegetation cover effects on ERS scatterometer data," IEEE Trans. Geosci. Remote Sens., vol. 37, no. 2, pp. 938-948, Mar. 1999.

[18] I. H. Woodhouse and D. H. Hoekman, "Determining land surface parameters from the ERS-1 wind-scatterometer," IEEE Trans. Geosci. Remote Sens., vol. 38, no. 1, pp. 126-140, Jan. 2000.

[19] M. Zribi, S. Le Hégarat-Mascle, C. Ottlé, B. Kammoun, and C. Guerin, "Surface soil moisture estimation from the synergetic use of the (multiincidence and multi-resolution) active microwave ERS wind scatterometer and SAR data," Remote Sens. Environ., vol. 86, no. 1, pp. 30-41, 2003.

[20] C. Prigent, I. Tegen, F. Aires, B. Marticoréna, and M. Zribi, "Estimation of the aerodynamic roughness length in arid and semi-arid regions over the globe with the ERS scatterometer," J. Geophys. Res., vol. 110, no. D09205, pp. 1-12, 2005, DOI: 10.1029/2004JD005370.

[21] L. Jarlan, P. Mazzega, E. Mougin, F. Lavenu, G. Marty, L. Frison, and P. Hiernaux, "Mapping of Sahelian vegetation parameters from ERS scatterometer data with an evolution strategies algorithm," Remote Sens. Environ., vol. 87, no. 1, pp. 72-84, Sep. 2003.

[22] S. Zine, L. Jarlan, P.-L. Frison, E. Mougin, P. Hiernaux, and J.-P. Rudant, "Land surface parameter monitoring with ERS scatterometer data over the Sahel: A comparison between agro-pastoral and pastoral areas," Remote Sens. Environ, vol. 96, no. 3/4, pp. 438-452, 2005.

[23] M. Grippa and I. H. Woodhouse, "Validation of surface scattering models across large footprints for global scatterometer applications," IEEE Trans. Geosci. Remote Sens., vol. 40, no. 10, pp. 2229-2640, Oct. 2002.

[24] M. Zribi, N. Baghdadi, N. Holah, and O. Fafin, "New methodology for soil surface moisture estimation and its application to ENVISAT-ASAR multi-incidence data inversion," Remote Sens. Environ., vol. 96, no. 3/4, pp. 485-496, Jun. 2005.

[25] T. D. Wu, K. S. Chen, J. Shi, and A. K. Fung, "A transition model for the reflection coefficient in surface scattering," IEEE Trans. Geosci. Remote Sens., vol. 39, no. 9, pp. 2040-2050, Sep. 2001.

[26] A. K. Fung, Microwave Scattering and Emission Models and Their Applications. Norwood, MA: Artech House, 1994.

[27] L. Rakotoarivony, O. Taconet, D. Vidal-Madjar, and M. Benallegue, "Radar backscattering over agricultural bare soils," J. Electromagn. Waves Appl., vol. 10, no. 2, pp. 187-209, 1996.

[28] M. Zribi, O. Taconet, S. Le Hégarat-Mascle, D. Vidal-Madjar, C. Emblanch, C. Loumagne, and M. Normand, "Backscattering behavior and simulation comparison over bare soils using SIRC/XSAR and ERASME 1994 data over Orgeval," Remote Sens. Environ., vol. 59, no. 2, pp. 256-266, 1997. 